\title{
A Survey on Voltage Dip Events in Power Systems
}

\author{
V. Barrera Núñez, J. Meléndez Frigola, and S. Herraiz Jaramillo
}

\begin{abstract}
A general view of voltage dip analysis is presented in this paper. The objectives to perform voltage dip analysis, activities inside of this analysis and methodological aspects are described from a data mining perspective. Basic data mining principles were taken as the basis to identify similar steps in power quality works involving classification and knowledge discovery tasks. This paper is centred in voltage dip event diagnosis in order to reduce the study and focus the analysis.
\end{abstract}

Index Terms - Fault location, voltage dip (sag), pattern classification, Power quality monitoring.

\section{INTRODUCTION}

$\mathrm{T}$ his article aims at surveying the techniques and methods used in the literature for knowledge discovery from registers of voltage dip event in power distribution networks.

\section{A. Voltage dip event: an electromagnetic phenomenon}

According to the IEEE 1159, a voltage event is considered a sag or dip when the RMS voltage decrease between 0.1 and 0.9 p.u of the nominal voltage and duration between half cycle and one minute. Therefore, this event is classified as a short duration variation.

The interest in analyzing, characterizing and classifying voltage dips is due to their impact on sensitive loads, causing damage which represents cost so much to the facilities [11]. Electronic equipment, such as Programmable Logic Controllers (PLC) or computers, may shut down, stopping a production or assembly line. The main causes of voltage dips are faults, induction motor starting, transformer energizing, load switching [6][12]. Hence, to diagnosis voltage dips it is necessary to extract hidden information of the registered current and voltage dip waveforms, such as: the fault location (point of origin), type of fault (single phase, three phase, etc) and determine the underlying causes that produced the event. In general, this extracted information will allow to assess the power quality supply with respect voltage dips and possibly will allow to predict future faulty behaviours too.

\section{B. The problem}

The importance of Power Quality monitoring has increased in the last years. This increment is due to electric open markets. The PQ is now focused on the high sensitivity of equipment and the energy supply continuity [1]. This phenomenon in the energy markets is caused

This work has been partilly supported by the Spanish government under the project DPI2006-09370 and ENDESA DISTRIBUCIÓN.

The authors are with the eXiT Group in the Institute of Informatics and Applications of the University of Girona, Spain, Girona, Campus Montilivi, 17071, e-mail: vbarrera@eia.udg.es, quimmel@eia.udg.es, sudden advances in the power quality monitoring devices. These devices have allowed facilities to register the electric parameters with better quality and quantity than devices of some years ago. These devices captured voltage and current waveforms, active and reactive powers, frequency, power factor and other information. The information is generated at several locations in local utility's service territory, asynchronously during long periods of time. This information is continuously monitored and registered, causing a great volume of data [2]. Consequently, the amount of data is increasing daily, due to the advances in the data storage technologies too. The end-user has a large amount of data to assess and reduce the power quality problems. The technologies to transform in a short time raw data in knowledge or answers have been few developed. Hence, the new goal of monitoring systems is to transform automatically this huge amount of data into useful knowledge in order to assist the management of the network.

In the context of PQ, several electromagnetic phenomena can be analysed. This paper is centred in voltage dip event diagnosis in order to reduce the study and focus the analysis. A review about the methods used to extract knowledge from dips registers is performed in this paper. These methods differ according the power quality monitoring goal but all of them follow data mining principles and steps [32]: (1) definition of the objectives for analysis, (2) selection, organisation and pre-treatment of data, (3) exploratory Analysis of data and subsequent transformation, (4) specification of the methods to be used in the analysis phase, (5) analysis of the data based on the chosen methods, (6) evaluation and comparison of the methods used and the choice of the final model for analysis, (7) interpretation of the chosen model and its subsequent use in the decision processes.

These basic steps have been taken as reference in the analysis of power quality papers dealing with dips classification and analysis.

\section{Antecedents: intelligent power quality monitoring}

The tendency of power quality monitoring to incorporate data mining mechanisms to automatically process raw has been enounced from the lasts nineties ([32] [33][34]). Instead of using the general concept of data mining, the concept intelligent power quality monitoring was used to design these forthcoming frameworks.

Typical objectives for dips monitoring are well know and described in the literature. For example in [33] the following requirements are pointed: characterisation of sag waveforms and its relation with pre and post event information, evaluation of causes of each event and location upstream or downstream from the monitoring point, analysis of load 
interaction issues, effect analysis based on existing equipment sensibility profiles (e.g. ITIC or SEMI curves), benchmarking based on statistical surveys or summaries and the evaluation of power conditioning equipment performance during events.

More recently, Ibraim and Morcos have surveyed works involving artificial intelligence and computational tools to assist power quality monitoring [35]. The survey basically reports a large list of references classified according to the technique involved: Expert systems, neural networks, fuzzy logic, genetic algorithms, wavelets and data compression.

\section{DATA MINING FOR ANALYSIS OF VOLTAGE DIPS}

In this section, the basic steps of data mining are used as guideline to survey the existing literature related with characterization and classification of voltage dips.

\section{A. Definition of the objectives for analysis}

In the literature three main objectives leading the analyze dip waveforms were found.

1) Objective 1: Determine the underlying cause or causes that led the voltage dip event: Papers trying to achieve this objective are few in comparison with those focused on the classification of power quality disturbances (dips, swell, harmonics, etc.). From our point of view it is very important the development of techniques to infer the underlying causes of an event from its register and knowledge on the power system. The difficulty in this development is because analysis of dips is usually done without taking into account additional information of the power system (typology of loads, incidences registered at the same date and time, firing protections, etc.). On the other hand major efforts have been put in the classification of disturbances [12].

A general classification of causes that produce voltage dip can be extracted from [12]: faults, induction motor starting, transformer energizing and load switching.

2) Objective 2: Determine the location of the voltage dip origin. This objective is treated under two perspectives: the first one tries to determine an accurate location of the voltage dip origin whereas in the second group of works, location is reduced to identify the propagation direction to determine the origin upstream or downstream with respect to the register equipment (relatively location).

a. Source accurate location: Since to obtain the accurate location it is necessary to know the network parameters such as: positive-negative and zero sequence impedance, installed loads, distances and conductor gauges among others. These parameters should allow to suitable modeling the power network and the model is used in the search of the dip origin. From network parameters and measured waveforms these methods [18] to [25] allow the researchers to know the node, section or a more accurate location where the fault is more probably located.

b. Source relative location: Here, the pinpoint fault location is not necessary. The effort is put in the deduction of a relative location upstream or downstream from the measuring point. The methods presented in [14] to [17] give this relative location from dip registers. In the group we include also direction methods. In [13] a comparison of four source relative location methods ([14] to [17]) with synthetic data is performed.

3) Objective 3: Assess the power quality. Voltage dip events are being used to evaluate the vulnerability of the industrial equipments and to define power quality indices. For example, dips duration and depth are depicted in the ITIC ${ }^{1}$ and CBEMA $^{2}$ curves to compare them with the sensitive curve of the equipments; the sensitive curves are supplied by the manufacturer of the equipment [44]. To achieve this objective it is not required to perform all data mining activities. However, selection, organisation and pretreatment of data before building the CBEMA and ITIC curves should be performed. A method to estimate the area of vulnerability due to voltage dip events in meshed network from ITIC curve is presented in [45].

Power quality campaigns are used to create power quality surveys and stochastic analysis of the network [4]. Results from these campaigns have been proposed to define power quality indices useful for: contractual purposes and assessment, comparison of power systems, reporting for system benchmarking purposes and for prioritizing of system maintenance and improvement investments. Others potential future applications based on signal processing to assess the power quality are listed in [48].

According to Dugan and McGranaghan [49], there are two streams of power quality data assessment, off-line and online analyses. The off-line power quality data assessment is performed off-line at the central processing locations (large volumes of data), whereas the on-line data analysis is performed within the instrument itself for immediate information dissemination. One of the primary advantages of on-line data analysis is that it can provide instant message delivery to notify users of specific events of interest, users can then take immediate actions.

\section{B. Selection, organisation and pre-treatment of data}

Many studies with synthetic data have been performed by the researchers. This situation is comprehensible due to the difficult to obtain raw data [12]. Also when raw data is available information richness is poor due to lack of variability on them. In this context, many works dealing with synthetic data avoid the tedious tasks of preparing, organizing and selecting (or filtering) the data to be used in the subsequent inference. This implies an additional problem in transferring research result obtained with synthetic data to the utilities since these steps have to be made when dealing with real and they are not trivial.

On the other hand, real data have origin diverse and typically organized in heterogeneous and distributed databases. Call center, power monitoring equipment and data bases from the network control center are typical sources of information useful for the power system diagnosis. Call centers reports costumer information related to faults and consequently it can be used to associate location (transformer, geographic situation) with disturbances, time of occurrence and affectation. Power quality monitors and other instruments with registering capabilities provide waveforms registers of events (three

\footnotetext{
${ }^{1}$ ITIC - Information Technology Industry Council

${ }^{2}$ CBEMA - Computer Business Equipment Manufacturers Association
} 
phase current and voltage sampled waveforms of dips). Sometimes, these waveforms are stored in a proprietary format difficult to be read without the appropriate software and other use standard formats as COMTRADE or comma separate values - CSV [36]. Unification in the same readable format is needed before starting the project. The new standard format for interchanging power quality data PQDIF $^{3}$ makes sharing of data between different types of monitoring systems much more feasible [49].

In the network control center a huge amount of useful information is stored. That is, all the registers reporting the network operation as switching, relays operation, reported faults and their location, maintenance actions, wheather conditions, underlying causes, etc. This type of information will be useful for both location and classification purposes if those registers can be associated with dip waveforms registered in substations, customers and other instrumentation equipment. An appropriate combination of all of this information has to be done to efficiently exploit the information systematically registered to achieve desired goals.

Consequently, the importance of pretreatment tasks when dealing with real data is cruzial and some specific tasks involved are the following:

1) Selection: Usually, the information about the registered disturbances can be extracted from different information data bases. Typically, call center database (information close to customer), register equipment database (power quality monitors, digital relays, etc.) and control center database of the utility. Different attributes contained in these different data bases can be useful for the power quality project. Selection of them depends on the relevance of them according to goals of the project and this relevance is not known. Correlation and sensitivity analysis have to be made in order to analyse the importance of attributes for specific goals.

Representative events have to be selected in order to cover the problem space. Also the most significant attributes describing the voltage dip have to be related to the problem to be solved. For instance, voltage perturbations with RMS voltage greater than 0.9 p.u of nominal voltage do not should be selected in a project of diagnosis of dips. The same selection has to be performed with other information contained in available data bases described before.

2) Organisation: It consists in defining an appropriate relational structure for the data being involved in the project based on the goals. In case of working only with dips it is necessary to think how different attributes of waveforms can be associated with its causes, origin or labels guiding the classification process. Depending on available information every waveform register, or sets of attributes extracted from the, could be associated with its underlying causes, typology of loads, the location of its origin, firing relays or other information. Indexing in a complete data base is needed when a large number of parameters is used instead of using flat structures.

3) Pre-treatment of data: Although this pretreatment has to be performed to all the attributes in order to detect outliers, corrupted data and missing values, when dealing with signals this pretreatment is associated to simple data

${ }^{3}$ PQDIF - Power Quality Data Interchange Format processing strategies to obtain a treatable representation. Is the case of obtaining the root mean square (RMS) sequence of three phase voltage and currents. Typically a sliding window of one cycle is used and a simple processing performed with the data contained in it. Typically the sliding window is updated each sample [4][6][29] to obtain a RMS waveform using the Short Fourier Transform (SFT). Similarly Discrete Wavelet Transform (DWT) can be applied to obtain different abstraction degrees of the waveforms. DWT is being used for better calculating the time related parameters, while the SFT for obtaining the magnitude related parameters [5]. In the next activity, these transformed signals are used to extract significant information.

\section{Exploratory Analysis of data and subsequent transformation}

This activity focused on the treatment of disp is used to calculate attributes extracted from time signals and RMS sequences. Authors refer to the output of this processing as: features, indices or descriptors [1][3][6]. These features are calculated to describe the behavior of the dip event waveforms. The features are defined in order to be representative of hidden information contained in waveforms. Therefore, a selection procedure has to be performed to take into account the representatives of those features with respect to desired goals.

Some authors propose a segmentation of the waveform to distinguish steady or stationary behaviours from the transients or non-stationary parts [12][30]. The purpose of the segmentation is to determine the signals processing techniques that can be applied in each segment. Three ways based on residuals models, RMS sequences and DWT are described in [30]. On the contrary, other signal segmentation method based on mathematical morphology and fractal technique in [39] is presented. Both segmentation methods [30][39] show good performance in accuracy and calculation speed.

Later, the Exploratory Data Analysis is performed with the existent data base in order to find out relations or common behaviors contained in the whole data base. The objective is to find out patterns and characteristics, which allow to establish relations between the data with the solution of the problem, so the extracted knowledge should allow to achieve any objective explained in the first activity described above, so information about the voltage dip event like: its underlying cause and event source location are found out from the selected features.

Some identified relevant features related with voltage dip analysis that are commonly used are the following:

1) Voltage sag magnitude on each phase $-S_{a}, S_{b}, S_{c}$ : The voltage sag magnitude $\left(\mathrm{S}_{\mathrm{a}}, \mathrm{S}_{\mathrm{b}}, \mathrm{S}_{\mathrm{c}}\right)$ is calculated in per unit of pre-fault voltage or nominal voltage [1] according to IEEE 1159.

2) Characteristic voltage - $V_{c}$ and Positive-Negative factor - PN factor $F$ : These two features contain information about the type and unbalance grade of the voltage dip, respectively [28][6][7]. These features are calculated with two algorithms: Six-phase and Symmetrical component. In [28] a detailed description about them is 
performed, the performance of each one is evaluated too.

3) Fundamental voltage component - $V_{l}$ : This feature is calculated through DFT. $\mathrm{V}_{1}(\mathrm{t})$ is useful to obtain the phase angle shift [6]. Some researchers use the fundamental voltage component magnitude $-\mathrm{V}_{1}$ as a feature, and other use the fundamental voltage as a function of time $-V_{1}(t)$ to obtain all the others features, it is possible if the harmonic level is low according to the recommended limits.

4) Phase angle shift - $\psi(t)$ : The fourth feature $(\psi)$ can be obtained from $V_{1}(t)$. A formula in function of $V_{1}(t)$ to obtain $\psi$ as a function of time in [6][29] is presented and explained.

5) Characteristic phase angle jump: The argument of the complex characteristic voltage $-\mathrm{V}_{\mathrm{c}}$. This features is use commonly to characterize voltage dips led by single-phase faults [46].

6) Loss of voltage $-L_{v}$ : $\mathrm{L}_{\mathrm{v}}$ is defined as the integral of the voltage drop during the event; the expression to obtain this feature in [6] is described and presented.

7) Sequence voltage - $V_{0}, V_{1}, V_{2}$ : The zero sequence voltage $-\mathrm{V}_{0}$ is very used to take advantage of its presence in unbalanced fault conditions. A fault location test using the three sequence voltage like features is performed in [37][38].

8) Frequency jump index - FJI: Is a function of maximum and minimum frequencies presents in $\mathrm{V}_{0}(\mathrm{t})$. These frequencies are computed using DWT. This feature is proposed in [7].

9) Ratio of the currents - $I_{s a g} / I_{s s}$ : Ratio between the fundamental frequency component of the currents during $\left(\mathrm{I}_{\mathrm{sag}}\right)$ and before $\left(\mathrm{I}_{\mathrm{ss}}\right)$ of voltage dip event. It can be used to determine the relative location of the event, an algorithm to identify the relative location and cause that led the voltage dip event in [40][41] is presented.

10) Second order harmonic current: This feature is useful to determine the transformer saturation voltage dip events [40][41]. The second harmonic component of the current is relatively large when the transformer is energized or the transformer voltage suddenly changes.

11) Difference of the active powers: The difference of the active power before and after induction motor starting is used to obtain the relative location in case of induction motor starting [40][41].

D. Specification of the methods to be used and Analysis of the data based on the chosen methods

The fourth and fifth data mining activities will be described together. From this activity a model based on the available data will be obtained. Then, it is important to select the appropriate technique according to the desired goals (location, classification, etc.). In order to achieve the first and second objective (defined in subsection A of this section), multiple methods have been used:

1) Strategies used by the researcher to achieve the objective 1 - Underlying cause: In the literature few papers about treatment of dips to infer the underlying causes have been found. A methodology to determine the cause that produces a voltage dip event in [40][41] is presented and tested. The methodology is able to discriminate if the dip is led for an induction motor, transformer or line fault. Moreover, the relative source location of the perturbation is given too (upstream or downstream of the measure point). With the ratio of the pre-fault and fault current the line fault cause is determined. The starting motor cause is determined through the increasing of active power, and the energizing transformer cause from the second harmonic component of current [40][41].

2) Strategies used by the researchers to achieve the objective 2 - Source location: According to the objectives described in the section II, the dip source location results are given in two ways, so accurate or relative location is obtained. The source relative location will not be described here, because the relative location through methods proposed in [14] to [17] is being obtained, which from voltage and current waveforms determine the relative location, hence the data mining is not require to obtain it. The accurate source location methods for the same reasons will not be described either, [18] to [25].

Three general strategies are being used by the researchers to find out the accurate location of the voltage dip source.

$i$. Using deterministic and statistic classifiers: This is the strategy widely used to classify power system events. When the amount data is limited, the deterministic classifiers are used; on the contrary, if the amount of data is large, the statistical classifiers are convenient use them [12].

The voltage dip statistical classifiers are conformed by one or combination of techniques based on statistical learning theory, for instance: Artificial Neural Networks ANN (RBF), Support Vectorial Machine - SVM, Learning Algorithm Multivariable Data analysis - LAMDA and Finite Mixtures [8].

The classifiers based on radial basis function (RBF) have taken over from classic ANNs [9][10]. SVM is a new network that implements a special training algorithm that maximizes the separating margin between two classes. The two main issues of interest in SVM classifiers are the generalization performance and the complexity of classifier which is a practical implementation concern [12]. Therefore, SVM networks have advantages with respect to ANNs [9]. These networks are used in [9][10][12] to classify power quality events, demonstrating ability to recognize voltage dips and others events. A SVM classifier able to discriminate between five types of dips is presented and analyzed in [12]. The proposed SVMs use Gaussian RBF kernels and it was training and tested with registered and synthetic waveforms of two different networks. To characterize each waveform 72 features was used, 60 features related with RMS sequences and 12 related with the harmonic distortion.

LAMDA is a technique that uses fuzzy classification taking advantage of fuzzy logic and hybrid connectors. It has been used to determine fault location in power distribution systems. A hybrid methodology based on LAMDA and Ratan Das fault location method [18] to estimate the type and location of the fault in [1] is described and tested. The methodology consists in extract features of the current and voltage dip events, after project these 
features in their principal components - Principal Components Analysis and use these projections as input data of five LAMDA nets which locate the fault in a power distribution system (one single-phase net, two phase-phase nets and two three-phase nets). The output of LAMDA and Ratan Das method to improve the fault location estimation is correlated, this combination allow to reduce the multiple estimation.

From the extracted patterns of the synthetic current and voltage dip events, a statistical model to determine the location of fault is built in [8]. This methodology is based on Finite Mixture distributions and uses the maximum depth of the dip event in each phase and the respective current values, hence six features characterize each synthetic dip event.

The voltage dip deterministic classifiers most common used are: expert system and fuzzy logic. A comparison between a deterministic classifier based on expert system and statistical classifier based on SVM is presented in [12]. The advantages and drawbacks of each one in the power system event classification is described. A fuzzy classifier to detect and classify power system events (flicker, impulse, swell, dip, notch, transient and harmonic) in [27] is described. Based on eleven features and fifteen fuzzy rules different types of event was distinguished by the fuzzy classifier.

ii. Making matching data or features: This strategy consists in matching extracted features from registered data and extracted synthetic feature from simulation tools. For example, in [3] by matching voltage and current signals obtained by recording devices and PSS/E software, the dip source location is found out through the system by utilizing an iterative searching process (optimization algorithm based on genetic algorithms). The source location will be determined by the simulated signals that best match the recorded ones.

A similar test in [37] and [38] taking like features the positive-negative and zero sequence voltages is presented, so a comparison between registered voltages and generated voltages is performed in order to determine the dip source location. The synthetic sequence voltages were obtained from dip perturbation waveforms performed in all buses of the power system.

iii. Using the network topology continuously: The techniques classified in this group using the network topology constantly to determine the dip source location. The difference between this group with the first and second group is that the others use the network topology one time; due to these need the network topology to generate the synthetic data only. The strategies in this group estimate the source location from a tree that represent the network topology, so synthetic data do not required for these strategies.

A new algorithm to locate PQ event source in [42] is presented. This algorithm is able to determine the line where possibly is the dip source location. From the impedance $(\mathbf{Z})$, coverage and direction matrix this method estimate the source location. The coverage matrix associates the register equipments with each line of the network. The direction matrix contains the relative source location (upstream or downstream) of the dip event respect to each register equipment. This direction from the method described in [14] is obtained. The ambiguity in the perturbation source location; due to multiple estimation is reduced by the distance calculation. However, the computed distance for perturbations so far the measurement point is not accurate; because of the distance calculate algorithm does not take into account the leakage current.

Similarity, other new algorithm to determine the voltage dip source location is presented in [43]. A procedure with ten steps is described and tested using the IEEE 30-bus benchmark system. In general the busses with severe voltage dips are close to the fault location and the current change on each branch connected to these busses significantly increases when the dip event occurs. Based on this fact, from a deviation index of each branch current change before and after the voltage dip event the procedure estimate the dip event source location. This procedure obtains the source location without computed the direction of the perturbation.

The method planted by Dong [42] is for radial distribution power networks, while the procedure proposed by Chang [43] is for both radial and meshed power networks. This is a drawback to the first method because the source location algorithm in meshed network is few developed.

Like was mentioned in the previous section, others methods based on in the network parameters are using to determine the accurate fault location [18] to [25].

The activities six and seven will not be described due to their nature, that is, they propose an evaluation, comparison and interpretation of the chosen methods and the selected method respectively.

\section{CONCLUSION}

Nowadays, the main objectives which the researchers are analyzing voltage dip were identified, and are three: the first, determine the underlying cause or causes that led the voltage dip event. The second objective, determine the location of the voltage dip origin, and third, assess the power quality with maintenance and benchmarking purposes.

In the first objective, the classifiers commonly used to extract knowledge of the voltage dip events are: SVM, ANN of radial-basis function, LAMDA, Fuzzy logic, Expert systems and Finite mixture distributions. The most papers found out in the literature are centered on statistical techniques, especially in ANNs. With few exceptions, the most papers propose methodologies to discriminate between the different power quality perturbations (harmonic, swell, dip, flicker, etc) and do not propose methodologies to determine the underlying causes that led a voltage dip event, such as: motor starting, transformer energizing or network faults.

To decrease the multiple estimation introduced by last methods ([18] to [25]) in the fault location, the LAMDA [1] technique and Finite Mixtures distributions [8] are being used. The multiple estimation is reduced because these techniques allow to know the zone where possibly is located the fault.

Due to the difficult to acquire raw data, the most studies with synthetic data are being performed. The use of synthetic data should avoid because reduce the applicability of the resulting classifier. 


\section{REFERENCES}

[1] J. Mora, V. Barrera, G. Carrillo, "Fault Location in Power Distribution Systems Using a Learning Algorithm for Multivariable Data Analysis". IEEE Transactions on power delivery, vol. 22, no. 3, July 2007, pp. 1715-1721.

[2] S. Santoso, J. Lamoree, "Power quality data analysis: from raw data to knowledge using knowledge discovery approach", Procee. of the IEEE Pow. Engin. Soc. Trans. and Distr. Conf., vol. 1, pp. 172-177, 2000 .

[3] M. Kezunovic, Y. Liao, "Fault Location Estimation Based on Matching the Simulated and Recorded Waveforms Using Genetic Algorithms", Dev. in Pow. Syst. Prot., Conf. Public. No. 479, IEE 2001, pp. 399-402

[4] M. H. J. Bollen, "Understanding Power Quality Problems: Voltages Sags and Interruptions". New York: IEEE Press, 2000.

[5] M. Kezunovic, "Automated Analysis of Voltage Sags, their Causes and Impacts", Power Engineering Society Summer Meeting, 2001. IEEE, pp. 1113-1117.

[6] M. Bollen, D. Sabin, "International Coordination for Voltage Sag Indices”, PES TD 2005/2006, May 21-24, 2006, pp.:229 - 234

[7] R. Mukerjee, B. Tanggawelu, G. Rogers, "Fuzzy Clasiffication Based Identification of voltage Sag Via Wavelets", Procee. of the $9^{\text {th }}$ Intern. Conf. on neural Infor. Proces. ICONIP'02, vol. 5, pp. 2381-2385, 2002.

[8] J. Cormane, H. Vargas, G. Ordóñez, "Fault Location in Distribution Systems by Means of a Statistical Model", Trans. \& Distr. Conf. and Exposition: Latin America, 2006. TDC '06. IEEE/PES, 2006, pp.1-7.

[9] P. Janik, T. Lobos, "Automated Classification of Power-Quality Disturbances Using SVM and RBF Networks", IEEE Transactions on Power Delivery, vol. 21, no.3, July 2006, pp. 1663-1669.

[10] C. Duarte, V. Vega, G. Ordóñez, "Automatic Power Quality Disturbances Detection and Classification Based on Discrete Wavelet Transform and Artificial Intelligence", TDC '06. IEEE/PES, 2006.

[11] M. McGranaghan, B. Roettger, "Economic evaluation of power quality". IEEE Power Engineering Review, vol.22, no. 2, pp. 8-12, 2002.

[12] M. Bollen, I. Gu, P. Axelberg, E. Styvaktakis, "Classification of Underlying Causes of Power Quality disturbances: Deterministic Versus Statistical Methods", Research article, EURASIP Journal on Advances in Signal Processing, vol 2007, article ID 79747, 17 pages.

[13] C. Leborgne, D. Karlsson, J. Daalder, "Voltage Sag Source Location Methods Performance Under Symmetrical and Asymmetrical Fault Conditions", Transmission \& Distribution Conference and Exposition: Latin America, IEEE/PES, pp: 1-6, August, 2006.

[14] A. Parson, W. mack, E. Powers, J. Soward, "A Direction Finder for Power Quality disturbances based Upon disturbance Power and Energy", IEEE Transactions on Power Delivery, vol. 15, no. 3, pp. 1081-1086, July 2000.

[15] T. Tayjasanant, C. Li, W. Su, “A Resistance Sign-Based Method for Voltage Sag Source Detection", IEEE Transactions on Power Delivery, vol. 20, no. 4, pp. 2544-2551, October 2005.

[16] A. Kumar, A. Routray, “Ap plying Distance Relay for Voltage Sag Source Detection", IEEE Transaction on Power Delivery, vol. 20, no. 1, pp.529-531, January 2005.

[17] C. Li, T, Tayjasanant, W. Su, X. Liu, "Method for Voltage-sag-source Detection by Investigating Slope of the system Trajectory", IEE Proce. Gener. Trans. and Distr., vol. 150, no. 3, pp. 367-372, 2003

[18] R. Das, "Determining the locations of faults in distribution systems", Ph.D. dissertation, Univ. Saskatchewan, Saskatoon, SK, Canada, 1998.

[19] D. Novosel, D. Hart, and J. Myllymaki, "System for locating faults and estimating fault resistance in distribution networks with tapped loads", U.S. Patent 5839 093, 1998.

[20] R. K. Aggarwal, Y. Aslan, and A. T. Johns, "An interactive approach to fault location on overhead distribution lines with load taps," in Proc. Inst. Elect. Eng., Develop. Power Syst. Protection, 1997, pp. 184-187,Conf. Publ. 434.

[21] L. Yang and C. Springs, "One terminal fault location system that corrects for fault resistance effects," U.S. Patent 5773 980, 1998.

[22] J. Zhu, D. Lubkeman, and A. Girgis, "Automated fault location and diagnosis on electric power distribution feeders," IEEE Trans. Power Del., vol. 12, no. 2, pp. 801-809, Apr. 1997.

[23] S. Krishnaswamy and A. St-Jacques, "A new fault location algorithm for radial transmission lines with loads," IEEE Trans. Power Del., vol. 4, no. 3, pp. 1676-1682, Jul. 1989.

[24] M. S. Choi, S. Lee, D. Lee, and B. Jin, "A new fault location algorithm using direct circuit analysis for distribution systems," IEEE Trans.Power Syst., vol. 19, no. 1, pp. 35-41, Jan. 2004.
[25] A. Girgis, C. Fallon, and D. Lubkerman, “A fault location technique for rural distribution feeders,” IEEE Trans. Ind. Appl., vol. 26, no. 6, pp. 1170-1175, Nov./Dec. 1993.

[26] C. Leborgne, R. Karlsson, "Voltage Sag Source Location Methods based on voltage measurements only", Submitted to IEEE Transactions on Power Delivery, TPWRD-00715-2006.

[27] M. Kezunovic, Y. Liao, "A novel software implementation concept for power quality study", IEEE Transactions on Power Delivery, vol. 17, no. 2, pp. 544-549, 2002.

[28] M. Bollen, "Algorithms for Characterizing Measured three-Phase Unbalanced Voltage Dips", IEEE Transactions on Power Delivery, vol. 18 , no. 3 , July 2003 .

[29] Voltage sag indices draft 2, working document for IEEE P1564, December 2001.

[30] M. Bollen, I. Gu, "Signal Processing of Power Quality Disturbances", IEEE Press Series on Power Engineering, 2006.

[31] G. Zheng, X. Yan, H. Li, D. Liu, "Classification of voltage Sag Based on Wavelet Transform and Wavelet Network", Proceedings of the Third Conference on Machine Learning and Cybernetics, Shangai, August 2004.

[32] Giudici P, “Applied Data Mining. Statistical Methods for Business and Industry", Wiley 2003.

[33] McGranahan M, "Trends in Power Quality Monitoring", IEEE Power Engineering Review, October 2001.

[34] McEachern A, "Roles of Intelligent Systems in Power Quality Monitoring: Past, Present, and Future" in Intelligent System Applications to PQ Monitoring Systems" Panel Session IEEE Summer Meeting, pp: 1103-1105, 2001.

[35] Ibrahim, W., Morcos M. "Artificial Intelligence and Advanced Mathematical Tools for Power Quality Aplications: A Survey", IEEE Transactions on Power Delivery, Vol 17, $\mathrm{n}^{\circ} 2$, April 2002.

[36] M. Kezunivoc, I. Rikalo, "Automating the Analysis of Faults and Power Quality", IEEE Computer Applications in Power, pp. 46-50, January 1999.

[37] W. Nazmy, W. Mahmood, R. Mukerjee, V. Ramanchandaramurthy, "Fault Point identification in a Power Network Using Single-Point Measurement", TENCON IEEE Region 10 Conference, vol. 3, pp. 381-384, Novembre 2004.

[38] -, "Identification of Voltage Sag Origin in a Measurement Deficient power Network", National Power and energy Conference - PECon 2003 Proceedings, pp. 347-351, 2003.

[39] G. Li, M. Zhou, Y. Luo, Y. Ni, "Power Quality Disturbance Detection Based on Mathematical Morphology and Fractal", IEEE/PES Transmission and Distribution Conference \& Exhibition: Asia and pacific, pp. 1-6, 2005.

[40] Seon A., Dong W., Li C., Seung M., "Determination of the Relative Location of Voltage sag source According to event Cause", Power Engineering Society General Meeting, 2004. IEEE June 2004 pp: 620 - 625 vol. 1.

[41] Kyoung K., Jin P., Jong L., Seon A., Seung M., "A Method to determine the Relative Location of Voltage Sag Source for PQ Diagnosis", ICEMS 2005, Proceedings of the Eighth International Conference Volume 3, 27-29 Sept. 2005 pp::2192 - 2197.

[42] Dong W., Li C., Joong K., Seung M., Jang S., Jong C., “A New Algorithm to Locate Power-Quality Event Source With Improved Realization of Distributed Monitoring Scheme", IEEE Transactions on Power Delivery, vol. 21, no. 3, July 2006.

[43] G. Chang, J. Chao, C. Chen, "A New Procedure for Tracking the Source Location of Voltage Sags", Power Engineering Society General Meeting, 2007. IEEE, pp. 1-4, June 2007.

[44] Geun-Joon L., Mihaela A.,Gerald-Thomas H., "A Power Quality Index Based on Equipment Sensitivity, Cost, and Network Vulnerability", IEEE Transactions on Power Delivery, vol. 19, no. 3, pp. 1504-1510, July 2004.

[45] Chang-Hyun P., Gilsoo J., "Stochastic Estimation of Voltage Sags in a Large Meshed Network", IEEE Transactions on Power Delivery, vol. 22, no. 3, pp. 1655-1664, July 2007.

[46] Lidong Zhang; Bollen, M.H.J, "A method for characterisation of three-phase unbalanced dips (sags) from recorded voltage waveshapes";

Telecommunications Energy Conference. pp.:9. 6-9 June 1999.

[47] Research Article

[48] M. McGranaghan; S. Santoso, "Challenges and Trends in Analyses of Electric Power Quality Measurement Data", EURASIP Journal on Advances in Signal Processing, vol. 2007, article ID 57985, 5 pages.

[49] R. Dugan, M. McGranaghan, S. Santoso, H. Wayne, "Electrical Power Systems Quality, Second Edition”, United Kingdom, McGraw - Hill, 2007. 